PharmacoEconomics \& Outcomes News 877, p20 - 1 May 2021

\title{
Specialty and cancer drugs set to drive US drug expenditure in 2021
}

Compared with 2019, pharmaceutical expenditures during 2020 increased by $4.9 \%$ in the USA, equivalent to a total of $\$$ US535.3 billion, and in 2021, overall prescription drug expenditure is projected to increase by $4 \%-6 \%$, driven primarily by specialty and cancer drugs and the ongoing expediture changes associated with the COVID-19 pandemic, according to an analysis published in the American Journal of Health-System Pharmacy.

The authors reviewed historical trends in pharmacutical spending for 2020 and previous years using data from the IQVIA National Sales Perspective database, focusing in particular on non-federal hospitals and clinics. Data for pipeline drugs were obtained from IPD Analytics clinical pipeline database. The impact of the COVID-19 pandemic on drug spending was examined as one of several additional analyses.

In 2020 , the drug expenditure increase of $4.9 \%$ was driven by changes in utilisation $(+2.9 \%)$ and new drugs $(+1.8 \%)$. Price changes had a minimal impact $(+0.3 \%)$. Drug spending increased by $8.1 \%$ in clinics, but decreased by $4.6 \%$ in non-federal hospitals - a result of reduced utilisation. Overall drug expenditure was greatest for adalimumab ( $\$ 24.9$ billion), apibaxan ( $\$ 12.8$ billion) and insuline glargine ( $\$ 9.7$ billion). In non-federal hospitals, three drugs commonly used for treating critical COVID-19 patients (tocilizumab, dexmedetomidine, and vasopressin) saw dramatic expenditure growth $(+263.3 \%$, $+103.3 \%$, and $+56.1 \%$, respectively); however, cancer drugs remained the main drug by expenditure, despite a reduction of $15.7 \%$. For 2021 , overal drug expenditure is expected to grow $7 \%-9 \%$ in clinics and by $3 \%-5 \%$ in non-federal hospitals. This will be driven mainly by cancer drug and biosimilar spending from new drug approvals and expanded indications. The ongoing rapid growth in specialty drug spending is expected to exceed $50 \%$ of overall drug expenditure in 2021. 